FORMATION Formation emploi

Revue française de sciences sociales

95 | juillet-septembre 2006

Pêle-mêle

\title{
Jeunes dans la fonction publique territoriale : entre gestion publique et marchande
}

Recruitment of young people by the French territorial civil service: between public and commercial practices

Rekrutierung Jugendlicher im Öffentlichen Dienst : zwischen privater und

öffentlicher Verwaltung

\section{Françoise Dauty et Philippe Lemistre}

\section{OpenEdition}

Journals

Édition électronique

URL : http://journals.openedition.org/formationemploi/2299

DOI : 10.4000/formationemploi.2299

ISSN : 2107-0946

Éditeur

La Documentation française

Édition imprimée

Date de publication : 30 septembre 2006

Pagination : 41-58

ISSN : 0759-6340

Référence électronique

Françoise Dauty et Philippe Lemistre, « Jeunes dans la fonction publique territoriale : entre gestion publique et marchande ». Formation emploi [En ligne], 95 | juillet-septembre 2006, mis en ligne le 30 septembre 2008, consulté le 30 octobre 2020. URL : http://journals.openedition.org/formationemploi/ 2299 ; DOl : https://doi.org/10.4000/formationemploi.2299

(c) Tous droits réservés 


\section{Secteur
d'activité}

\section{Jeunes dans la fonction publique territoriale: entre gestion publique et marchande}

par Françoise Dauty et Philippe Lemistre*

L'image du fonctionnaire recruté après un concours écrit puis titulaire à vie cache une grande diversité de situations. Comparée à la fonction publique d'État, la fonction publique territoriale recourt à de nombreux contractuels et utilise des modes de gestion proches du secteur marchand.

Les enjeux de recrutement face aux départs à la retraite pour les années à venir sont particulièrement forts (Germe, Monchatre et Pottier, 2003). Or, employeur d'un quart de la population salariée, la fonction publique joue un rôle important, tout particulièrement dans l'emploi des jeunes diplômés (Audier,1997). Elle ne constitue pas néanmoins un ensemble homogène. Elle se divise en trois grands ensembles, la fonction publique hospitalière, la fonction publique d'État et la fonction publique territoriale (respectivement FPE et FPT dans la suite du texte). Chaque fonction publique crée et gère ses emplois : par ministère au sein de la FPE, par filière et niveau hiérarchique pour la FPT, par filière et établissement en distinguant personnel réglementé ou non pour la fonction publique hospitalière. Au sein de chacune d'elles coexistent des pratiques de gestion différentes et des types d'emplois très divers.

Nous nous focaliserons ici tout particulièrement sur la fonction publique territoriale, notamment en la compa-
* Françoise Dauty est ingénieur de recherche CNRS au Lirhe (Laboratoire interdisciplinaire de recherche sur les ressources humaines et l'emploi). Ses principaux axes de recherche relèvent de l'économie de l'emploi et de l'éducation, des dimensions territoriale et institutionnelle (Analyse des relations entre formations et emplois et dynamiques territoriales ; évolution des modes de recrutement et de gestion des compétences et rôle des diplômes).

Philippe Lemistre est économiste au Lirhe, centre régional associé au Céreq. Ses travaux portent sur les carrières salariales et l'articulation entre système éducatif et marché du travail. II a récemment publié avec J.F. Giret, " Le déclassement à l'embauche des jeunes: vers un changement de la valeur des diplômes? ”, Brussels Economic Review, Special Issue in "Economics of Education and Human Ressources», Vol. 47, n 3, 2004. II coordonne un ouvrage avec J.M. Lattes et P. Roussel, Individualisation des salaires et rémunération des compétences, Économica, Collection "Recherche », à Paraître, septembre, 2006. 
rant à la fonction publique d'État ; la fonction publique hospitalière n'est pas retenue du fait de sa spécificité en termes d'emplois et de formations (emplois réglementés, filières de formations spécifiques).

Les emplois de la fonction publique territoriale ont connu une forte hausse ces dernières années, sous l'effet de la décentralisation notamment. Si la FPE reste le plus gros employeur, la «croissance de l'emploi public repose sur la FPT» (Raynaud, 2003). Cette dynamique dépend de plusieurs facteurs dont les effets se combinent, tels que l'étendue des services offerts et des compétences territoriales, les transformations des modes de gestion (externalisation, gestion associative) et des modes de recrutement écartelés entre renforcement de la professionnalisation et recrutement social ${ }^{1}$ (Le Lidec, de Montricher, 2004).

$\mathrm{Au}$ moment où émergent de nouveaux enjeux de performance liés à l'organisation et à la qualité des services rendus et où les transferts de compétences de l'État vers les collectivités territoriales s'intensifient, il apparaît opportun de s'intéresser à la diversité des situations nées de ces évolutions.

Les différences de modes de gestion et la nature des emplois offerts contribuent à distinguer les deux fonctions publiques. La gestion de la FPE est fondée sur le principe de «carrière » et le recrutement s'effectue principalement sur des critères d'âge ${ }^{2}$ et de diplôme. La FPT, quant à elle, intègre une dimension « emploi ». La gestion s'effectue dans des filières et cadres d'emploi et surtout au niveau des collectivités et non au plan national. La FPT combine une gestion des emplois de type publique (concours, statut) et marchande (procédure de recrutement, carrière dans l'établissement, pouvoir de l'employeur).

L'objectif de cet article est d'analyser ces spécificités de la fonction publique territoriale en examinant les premières années de vie active des jeunes salariés de la fonction publique territoriale et de la fonction publique d'État, trois ans après leur sortie du système éducatif. Il s'agit notamment de dépasser les appro-

${ }^{1}$ Le recrutement est qualifié de social quand ce sont des exigences de caractère social qui dominent, la volonté d'embaucher des populations défavorisées.

${ }^{2}$ Depuis le premier novembre 2005, les conditions d'âge pour l'accès aux concours de la fonction publique ont été supprimées dans la majorité des cas (DGAFP, ministère de la Fonction publique). ches habituelles qui relèvent souvent d'une vision dichotomique de type employeur public/privé.

Nous mobilisons l'enquête "génération 98 » du Céreq, qui s'intéresse aux parcours des jeunes ayant quitté le système éducatif en 1998, jusqu'à leur situation en $2001^{3}$.

Dans un premier temps, la spécificité de la fonction publique territoriale, en tant qu'employeur, est abordée dans le cadre d'une comparaison fonction publique territoriale / fonction publique d'État. Ensuite, les caractéristiques des jeunes employés par la FPT sont mises en regard de celles des jeunes de la FPE ; les modalités d'accès aux emplois et aux différents statuts sont examinées pour les deux fonctions publiques. En effet, si les caractéristiques individuelles des jeunes, notamment leurs caractéristiques scolaires, jouent un rôle décisif dans l'insertion professionnelle, les modalités de l'insertion sont porteuses et révélatrices des transformations structurelles des normes d'emploi liées ici au développement de l'emploi au sein de la FPT (Lefresne, Fondeur, 2000).

Le second temps concerne uniquement la FPT. Le rôle des caractéristiques individuelles et du type d'emploi occupé sur les salaires est précisé. Les types d'emplois font également l'objet d'une attention particulière afin de tenter d'identifier les caractéristiques associées aux différents statuts (emploi-jeune, contrat à durée déterminée - CDD, contrat à durée indéterminée - CDI, fonctionnaire). Il s'agit notamment de compléter les rares travaux réalisés sur l'évolution des emplois et des modes de gestion dans la FPT ${ }^{4}$.

\section{FONCTIONS PUBLIQUES D'ÉTAT ET TERRITORIALE : DES ENTITÉS PEU COMPARABLES ?}

La plupart des études économiques et statistiques abordent l'emploi public de manière assez globale,

\footnotetext{
${ }^{3}$ Pour une approche plus globale de " l'insertion des jeunes sortants 98 et la fonction publique », voir Di Paola V. et Moullet S. (2003).

${ }^{4}$ Notamment les travaux de l'Observatoire de l'emploi public (Rapport 2003, DGAFP, décembre 2003) ou bien ceux de l'Observatoire de la FPT, «Étude sur les non-titulaires dans les collectivités territoriales $», n^{\circ} 4$, septembre 2003.
} 
notamment celles qui traitent des salaires, en retenant une variable ou un critère de distinction unique, « secteur public» par opposition au secteur privé. Ceci suppose une certaine homogénéité des modes de rémunération et de recrutement au sein du secteur public qui s'opposerait à une grande diversité dans le secteur privé. Aborder le secteur public globalement suppose également une assez forte similarité entre les professions et les statuts. Or, toutes les études relatives à la structure des emplois comme aux modes de gestion soulignent la diversité qui les caractérise dans les trois fonctions publiques.

\section{Des différences dans les modes de recrutement et de gestion}

La première différence tient à la nature même de l'employeur public: d'un côté, un employeur « unique », l'État, qui fixe chaque année le nombre de postes ouverts aux concours; de l'autre, plus de 60000 employeurs qui ont une assez large autonomie pour recruter et manifestent une diversité de pratiques (26 régions, 100 départements, 36773 communes et 19000 EPL - Établissements publics locaux - et l'absence d'employeur au niveau national). En effet, si la FPT a été organisée en 1984 sur le modèle de la FPE, dans la pratique, les politiques de gestion du personnel l'ont éloignée du modèle statutaire initial (encadré 1).

La création de la FPT visait à donner aux collectivités une fonction publique professionnelle, construite sur le modèle de la fonction publique d'État. Aux difficultés juridiques qui sont apparues pour passer d'une multitude de statuts (antérieure aux lois de décentralisation) à un statut unique, s'ajoutent les débats qui ont accompagné cette construction d'un compromis entre la revendication d'autonomie des autorités territoriales pour le recrutement et la gestion de leurs agents et la transposition du modèle étatique. Ainsi la FPT, en permanente évolution depuis sa création, n'est pas une copie conforme de la FPE (Bourdon, 2004).

La gestion territoriale des emplois est une gestion décentralisée : les collectivités ont la liberté de créer et de supprimer des emplois. Cette liberté est encadrée (seuils de population pour certains emplois et règles de procédure à respecter), mais l'autorité territoriale garde le pouvoir de recruter et de nommer.
D'autre part, contrairement aux services de l'État, qui relèvent d'un employeur unique, les collectivités territoriales sont en concurrence entre elles pour le recrutement des agents.

La deuxième différence en matière de recrutement entre FPE et FPT, plus importante peut être, est liée à l'usage du concours. En effet, la fonction publique territoriale fait une utilisation originale du concours, qui ne constitue qu'une opération préalable au recrutement. Il conduit seulement à l'établissement de listes d'aptitude par ordre alphabétique et donc sans ordre de mérite. Il s'agit plus d'un examen avec numerus clausus. Surtout, et contrairement à ce qui se passe avec la FPE, il y a séparation relative entre les concours de recrutements nationaux, fonction d'un nombre de postes susceptibles d'être vacants, et ouverture de postes. Les lauréats du concours sont alors inscrits sur une liste d'aptitude (inscription pour une année, renouvelable deux fois) et peuvent se faire embaucher par les collectivités locales qui puisent dans ce vivier. Le recrutement proprement dit n'intervient qu'une fois la liste d'aptitude publiée, l'autorité locale demeurant libre de recruter qui elle veut sur la liste, ou personne. De fait, pour les cadres, de nombreux recrutements procèdent de sorte de bourses d'emplois qui consistent à publier des annonces de collectivités assorties des spécificités des postes; peuvent alors coexister des postes vacants et des lauréats sans affectation.

Enfin, une troisième distinction apparaît dans le recours plus large aux diverses formes d'emploi : gestion indirecte (associative, déléguée...), utilisation des mesures d'emploi (gestion sociale), large recours aux contractuels, possibilité de recruter directement (encadré 2) (Le Lidec, Montricher, 2004). Les personnels de catégorie $\mathrm{C}$, notamment, peuvent être recrutés directement sans concours. Les recrutements dans la FPT, y compris pour les emplois de fonctionnaires passant par la voie des concours, relèvent au final plus de procédures de recrutement classiques : affichage de profils de postes, entretiens, utilisation importante des réseaux. Le poids des recrutements directs conduit aussi les contractuels déjà en poste à passer les concours. "Les lauréats des concours externes de la FPT présentent un profil spécifique puisque 60 à $90 \%$ d'entre eux, selon les cadres d'emploi, sont déjà en fonction dans une collectivité » (Bourdon, 2004). Ainsi, la FPE sélectionne par 


\section{Encadré 1}

\section{Fonction publique territoriale et catégories d'emploi}

Les salariés de l'employeur public «territorial » relèvent de trois grandes catégories:

- Des fonctionnaires territoriaux : deux tiers de l'emploi territorial ;

- Des agents non titulaires de droit public, permanents ou non permanents, et les assistantes maternelles à domicile $(22 \%)$;

- Des emplois aidés : emplois-jeunes, CES, CEC (respectivement contrat emploi solidarité et contrat emploi consolidé, qui relèvent du droit privé), soit $\cong 10 \%$ (données CNFPT, Les agents non-titulaires au 1/1/2001, état des lieux, juin 20031.

Enfin, dans son acception large, la notion d'employeur territorial comprend les organismes publics (collectivités territoriales: communes, départements, régions et structures intercommunales), les établissements publics mais aussi des organismes privés (de type syndicats d'initiative, offices de tourisme, organismes d'action sociale, d'aménagement ou d'action sociale à vocation culturelle). Ces organismes emploient sous statut de droit privé. Il existe donc aussi des personnels sous statut de droit privé.

La fonction publique territoriale est organisée en huit filières qui correspondent aux grands domaines d'intervention des collectivités territoriales : administrative, technique, culturelle, sportive, sanitaire et sociale, sécurité, animation, sapeurs-pompiers.

Au sein de chaque filière, le statut de la fonction publique territoriale organise une hiérarchie entre les fonctionnaires en les répartissant en trois catégories /catégories existant aussi dans la fonction publique d'État).

La catégorie A correspond aux fonctions de conception et de direction. Le recrutement se situe au niveau de la licence, à l'exception de certains cadres d'emplois qui exigent des diplômes supérieurs. Les agents de la catégorie A ont vocation à être des cadres.

La catégorie B correspond à des fonctions d'application. Le recrutement se situe au niveau du baccalauréat à bac +2 . Les fonctionnaires de catégorie B peuvent encadrer des équipes, des ateliers, des services. Ils constituent un encadrement intermédiaire.

La catégorie C correspond aux fonctions d'exécution, nécessitant, pour certains métiers, des qualifications professionnelles spécialisées de type BEP/ CAP (brevet d'études professionnelles/certificat d'aptitude professionnelle).

Un fonctionnaire territorial est recruté sur un cadre d'emplois, au sein duquel il pourra exercer différents métiers. Chaque cadre d'emplois regroupe des agents dont les emplois et les métiers ont des caractéristiques professionnelles proches. Ils auront en commun des savoir-faire, des aptitudes, des connaissances, une formation, et des modalités de recrutement, de rémunération et d'avancement.

Dans les filières culturelle, médico-sociale, animation, sécurité et sport, le cadre d'emplois correspond souvent à un métier.

Exemples : le cadre d'emplois des attachés territoriaux regroupe aussi bien des chargés de communication, des juristes que des responsables du développement économique ou des directeurs des ressources humaines; un jardinier et un électricien peuvent tous les deux relever du cadre d'emplois d'agent de maîtrise de la filière technique.

Source : site CNFPT. 
concours à partir de niveaux de diplômes exigés, alors que la FPT s'assure, via le concours, d'un niveau avant d'effectuer un recrutement plus individualisé. Ce système pose en revanche pour les candidats le problème des « reçus-collés »; ainsi, des jeunes reçus aux concours peuvent ne pas trouver d'emploi et perdre le bénéfice de ce concours.

Le mode de recrutement de la FPT semble plus souple et devrait permettre en principe de mieux réaliser l'adéquation individu / emploi. Toutefois, l'obtention du concours ne garantit pas celle d'un emploi. En d'autres termes, dés l'entrée dans la FPT, le parcours semble moins sécurisé que dans la FPE, l'accès au marché interne étant nettement moins garanti. Rappelons qu'au sein des marchés internes, les individus bénéficient de carrières à moyen ou long terme assorties d'avantages sala- riaux qui peuvent avoir pour corollaire une mobilisation plus forte des salariés. Les marchés internes se distinguent du marché externe où les salaires sont déterminés plus fortement par les mécanismes de l'offre et de la demande et où la précarité est plus prégnante 5 . La FPT serait donc un exemple d'une combinaison du modèle marché interne (entrée à certains points et carrière) et marché externe via de nombreux contractuels et ses procédures de recrutement.

\begin{abstract}
${ }^{5}$ Si l'on se réfère à Doeringer et Piore (1971), le marché interne est une unité institutionnelle à l'intérieur de laquelle le prix et l'allocation du travail sont déterminés par des procédures administratives, par opposition au marché externe, où rémunération, formation et allocation du travail sont déterminées par les ajustements marchands.
\end{abstract}

\section{Encadré 2}

\section{Fonction publique territoriale et modes de recrutement $d^{\prime}$ 'agents non titulaires}

La voie normale d'accès à la fonction publique territoriale est le concours; cependant, certains recrutements dans la fonction publique peuvent intervenir sans concours.

Le recours à des agents non titulaires est encadré ; il ne peut en théorie s'effectuer que dans des cas limitativement énumérés.

Une collectivité peut recruter pour une courte période des non-titulaires pour :

- Des besoins saisonniers ou occasionnels ;

- Des remplacements de titulaires indisponibles (maladie, maternité ...).

Ces cas - contractuels non permanents - concernent $37 \%$ des agents non titulaires.

Elle peut, pour une période plus longue, CDD (contrat à durée déterminée) de 3 ans renouvelable, faire appel à des personnes ayant des compétences spécifiques :

- Lorsqu'il n'existe pas de cadre d'emplois correspondant ;

- Pour combler un emploi après un appel à candidatures de titulaires infructueux.

- Pour les emplois de "cabinet » et certains emplois de direction des grandes collectivités (régions, départements, villes de plus de 80000 habitants).

On trouve enfin d'autres types d'emplois: agents à temps non complet dans les petites communes, personnes handicapées, emplois fonctionnels de direction, emplois spécifiques.

L'ensemble de ces emplois représente $42 \%$ de non-titulaires permanents.

Enfin, les assistantes maternelles à domicile représentent $21 \%$ de ces emplois.

Les agents non titulaires permanents ont des caractéristiques assez particulières : plus de la moitié a plus de cing ans d'ancienneté, la part de la catégorie A est importante. Pour les non-titulaires non permanents, ce sont les emplois d'animateurs et d'agents d'animation qui sont principalement concernés. 


\section{Des profils différents}

Une comparaison descriptive entre les caractéristiques des sortants de la génération 98 en emploi dans la FPE et la FPT en 2001 (trois ans plus tard) témoigne très largement de cette distinction (tableaux 1, 3, 4). Toutefois, pour mieux saisir l'importance relative des éléments qui distinguent les deux fonctions publiques, la probabilité d'appartenir trois ans après la sortie d'études à la FPT a été estimée à partir d'un échantillon comprenant l'ensemble des salariés de la FPE et de la FPT (tableau 2 - procédure probit) ${ }^{6}$.

${ }^{6}$ L'ensemble de la population active n'a pas été retenu afin de saisir uniquement les différences entre FPE et FPT. D'autant que, pour être significative, la prise en compte du secteur privé, via un probit
L'échantillon complet comprend 6749 individus salariés en 2001, répartis en 5179 individus pour la FPE et 1570 pour la FPT, représentatifs (effectifs pondérés) de respectivement 21631 et 59845 jeunes. Soit un peu plus de $3 \%$ et de $10 \%$ de la génération 1998 en emploi en 2001, dont la majorité reste donc employée dans le secteur privé ou hospitalier public. Ces chiffres peuvent être comparés au total des populations en emploi, parmi lesquelles FPT et FPE représentent $6 \%$ et $10 \%$ de l'emploi global en $2001^{7}$. Ce constat peut paraître paradoxal puisque, comme cela

multinomial par exemple, aurait nécessité des distinctions au moins sectorielles ou par taille d'entreprise.

${ }^{7}$ Source DGAFP, La fonction publique de l'État, Rapport annuel 2002.

Tableau 1

Caractéristiques des jeunes employés par la fonction publique territoriale et la fonction publique d'État (en \%)

\begin{tabular}{|l|c|c|}
\hline & Fonction publique territoriale & Fonction publique d'État \\
\hline Age moyenne & 22 & 24 \\
\hline Femme $\%$ & 59 & 58 \\
\hline Diplômes $\quad \%$ & 5,4 & 2,3 \\
\hline Non-diplômés & 5,6 & 1,5 \\
\hline CAP-BEP non-diplômés & 6,1 & 1,8 \\
\hline CAP & 11,8 & 4,9 \\
\hline BEP & 3,4 & 2,1 \\
\hline Bac. non diplômé & 6,9 & 4,2 \\
\hline Bac pro. & 3,5 & 2,8 \\
\hline Bac. Techno. & 8,7 & 13,9 \\
\hline Bac géné-DEUG non Dip. & 6,9 & 5,2 \\
\hline BTS-DUT non Dip. & 5 & 4,1 \\
\hline DEUG & 11,4 & 7,1 \\
\hline BTS & 2,3 & 2,1 \\
\hline DUT & 6,1 & 11,2 \\
\hline Bac. + 3 & 5,8 & 7,9 \\
\hline Bac. + 4 & 7,3 & 8,4 \\
\hline Bac. + 5 & 0,8 & 10,3 \\
\hline IUFM échec & 2,9 & 10 \\
\hline Écoles et Prépa. & & \\
\hline
\end{tabular}

Sigles : Cap : certificat d'aptitude professionnelle ; BEP : brevet d'études professionnelles ; BTS : brevet de technicien supérieur ; DUT : diplôme universitaire de technologie ; DEUG : diplôme d'études universitaires générales ; IUFM : Institut universitaire de formation des maîtres. Lire : les détenteurs d'un BTS représentent 11,4\% des jeunes de la génération 98 employée au sein de la FPT en 2001, contre 7,1 \% pour ceux de la FPE. Source : Enquête Génération 98, Céreq. 
a été évoqué précedemment, la FPT recrute proportionnellement plus que la FPE. En fait, les recrutements de la FPT s'adressent davantage que pour la FPE à une population déjà sur le marché du travail, c'est-à-dire au-delà des trois premières années de vie active retenues ici. Cette hypothèse est largement confirmée par les modes de recrutement observés.

Les niveaux de diplômes sont clairement différenciés ; un tiers des recrutés de la FPT ne possède pas le baccalauréat contre seulement près de $13 \%$ dans la FPE. À l'inverse, presque la moitié (47,6\%) de jeunes recrutés dans la FPE a un diplôme égal ou supérieur à bac +3 ; ils ne sont que moins d'un quart $(23 \%)$ dans la fonction publique territoriale. La présence de nombre d'enseignants et de plus de cadres au sein de la FPE (tableau 4) explique évidemment, en grande partie, cet écart. Il faut garder à l'esprit que pour l'ensemble des comparaisons entre les deux fonctions publiques, les effectifs de la FPT représentent moins d'un tiers de ceux de la FPE. En conséquence, une part relative plus importante pour une catégorie donnée au sein de la FPT ne reflète pas une plus forte probabilité

Tableau 2

Probabilité d'appartenir à la fonction publique territoriale (FPT) en regard de la fonction publique d'Etat (FPE) (1)

\begin{tabular}{|c|c|c|c|}
\hline & FPT/FPE & & FPT/FPE \\
\hline Statut & & Diplômes & \\
\hline Fonctionnaire & ref. & non diplômés & réf. \\
\hline Contrat à durée indéterminée & ++ & CAP-BEP non diplômés & + \\
\hline Contrat à durée déterminée & ++ & CAP & $+(4)$ \\
\hline Contrat emploi solidarité & + & BEP & $\sim$ \\
\hline Emploi-Jeune & $+++(2)$ & Bac. non diplômé & $\sim$ \\
\hline Age & - & Bac Pro. & $\sim$ \\
\hline Femme & +++ & Bac. Techno. & -- \\
\hline Parcours & & Bac géné-DEUG non Dip. & $\cdots$ \\
\hline mois au chômage & - & BTS-DUT non Dipl. & -- \\
\hline mois en inactivité & $\cdots(3)$ & DEUG & -- \\
\hline mois en formation & - & BTS & - \\
\hline mois en études & -- & DUT & -- \\
\hline \multirow[t]{2}{*}{ temps accès au premier emploi } & $\sim$ & Bac. +3 & $\cdots$ \\
\hline & & Bac. +4 & $\cdots$ \\
\hline premier emploi = emploi actuel & +++ & Bac. +5 & $\cdots(4)$ \\
\hline premier emploi privé & + & Écoles et Prépa. & $\cdots$ \\
\hline Temps complet & $\cdots$ & & \\
\hline
\end{tabular}

\section{Lecture :}

(1) La régression logistique permet d'estimer la probabilité d'être salarié de la FPT à trois ans en fonction des différentes variables. L'effet de chaque caractéristique est estimé « toutes choses égales par ailleurs ». Lorsque le modèle comprend plusieurs variables continues, les probabilités doivent être recalculées pour chaque valeur ou combinaison des valeurs entre elles (pour différentes situations de référence). Compte tenu de la difficulté à choisir une situation plutôt qu'une autre, la méthode retenue ici est de comparer les variables de même type entre elles.

(2) Lire : la probabilité d'être en emploi-jeune est plus forte dans la FPT que celle d'être fonctionnaire par rapport à la FPE. Le fait d'être en emploi-jeune augmente cette probabilité d'appartenir à la FPT (ref. FPE) à peu près trois fois plus que le fait d'être en CES.

(3) Lire : avoir connu un mois d'inactivité pendant les trois premières années de vie active diminue la probabilité d'être employé par la FPT plutôt que par la FPE, ceci trois fois plus qu'un mois de chômage.

(4) Lire : la probabilité d'appartenir à la FPT plutôt qu'à la FPE est plus forte lorsque le jeune a un CAP que lorsqu'il est non diplômé. En revanche, par rapport aux non-diplômés, cette même probabilité est diminuée trois fois plus (que l'augmentation pour le CAP) lorsque le jeune a un bac +5 .

Source : Enquête Génération 98, Céreq. 
d'appartenir à celle-ci plutôt qu'à la FPE. Au sein de la seule FPT, un type de diplôme peut donc être proportionnellement plus représenté que dans la FPE, alors que les détenteurs de cette certification sont plus nombreux dans celle-ci.

En d'autres termes, un poids relatif de certains diplômés plus important dans la seule FPT n'a pas nécessairement pour corollaire un nombre absolu de jeunes détenteurs de cette certification supérieur à celui de la FPE. Par exemple, les BTS (brevet de technicien supérieur) représentent $11,4 \%$ des diplômés de la FPT contre $7,1 \%$ de la FPE (tableau 1). Mais compte tenu des effectifs relatifs, ces derniers sont évidemment plus nombreux au sein de la FPE (11,4\% de 1570 est inférieur à 7,1\% de 5 179).

Ceci explique que la probabilité d'appartenir à la FPT en regard de la FPE soit diminuée pour les titulaires de ce diplôme même s'ils sont, en proportion, plus nombreux dans la FPT (tableau 2). En termes de formation, la distinction la plus nette concerne le recrutement plus marqué au sein de la FPT des détenteurs des niveaux de certifications les moins élevés : les non-diplômés, les CAP (certificats d'aptitude professionnelle) et BEP (brevet d'études professionnelles). À tous les niveaux, on note une préférence pour les diplômes professionnels. Cependant, compte tenu de la remarque précédente, seul le CAP conduit à une probabilité plus élevée d'être recruté dans la FPT que dans la FPE. Les autres diplômes relativement bien représentés au sein de la FPT sont les baccalauréats professionnels et les BTS. Les jeunes diplômés des formations les plus professionnalisées sont donc les plus représentés au sein de la FPT. Les sortants de formations générales sont proportionnellement nettement moins nombreux que dans la FPE (tableau 1 : baccalauréats généraux, bacs +3 et 4 , Écoles et prépa.) ${ }^{8}$.

\section{Des modes d'accès à l'emploi distincts}

Les jeunes salariés en 2001 dans la FPT ont en moyenne connu quatre mois de chômage dans leur

\footnotetext{
${ }^{8}$ L'aspect dominant des formations professionnelles ou techniques est renforcé par des pourcentages proches au sein de chaque FP pour les bacs technologiques, les DUT (diplôme universitaire de technologie) et les bac +5 qui ont un contenu plus « professionnalisant » que les bac +3 et 4 notamment.
}

parcours depuis leur sortie du système éducatif contre deux mois et demi pour les salariés de la FPE (tableau 3). Trois raisons peuvent être avancées pour expliquer cette différence. La première est liée au niveau d'éducation, les difficultés d'insertion étant plus importantes pour les moins diplômés, relativement plus nombreux au sein de la FPT. En effet, si l'on compare les durées de chômage par niveau de diplôme, on constate que la différence FPE/FPT est surtout importante pour les diplômes supérieurs à bac +3 (de 1,6 à 4 mois). De plus, pour les diplômes inférieurs au baccalauréat, les écarts persistent $(0,4$ à 1,5 mois). Cette explication est en tout cas loin d'être suffisante. Deux autres explications sont plus plausibles; la première est liée à la procédure de recrutement par concours. Comme cela a été évoqué précédemment, dans la FPT il existe un temps de latence entre la réussite au concours et l'accès à un emploi. Ceci est particulièrement vrai pour les plus hauts niveaux de certification et peut expliquer le nombre de mois de chômage relativement important. Une autre hypothèse repose sur l'idée que la FPT pourrait être un second choix pour des jeunes sans emploi. Cette conjecture semble confirmée par un nombre de mois en chômage nettement plus conséquent, notamment pour les bac +4 . Ces diplômés ont connu en moyenne deux mois de chômage quand ils intègrent la FPE contre près de cinq mois pour la FPT. Ces explications peuvent se conjuguer : les jeunes en recherche d'emploi dans une zone déterminée, inscrits au chômage, peuvent passer un concours de la FPT entre autres démarches et rechercher indifféremment un emploi dans une collectivité territoriale ou dans le secteur privé. Les deux résultats suivants confortent cette hypothèse.

L'emploi occupé trois ans après la sortie du système éducatif est plus souvent un premier emploi dans la FPT (pour $43 \%$ des individus) que dans la FPE, même «toutes choses égales par ailleurs » (tableau 2). Or, l'accès à un emploi de la FPE est moins rapide quand l'emploi en 2001 est aussi le premier emploi, avec plus d'un mois d'écart avec la FPT. De plus, le nombre de mois en chômage est plus conséquent pour ces individus qui n'ont connu qu'un seul emploi, de quatre mois et demi contre toujours deux et demi pour les jeunes qui accèdent à la FPE.

Ces constats soulignent une autre caractéristique des emplois de la FPT : ils sont, surtout les moins qualifiés, plus proches des emplois du secteur privé que 
Tableau 3

Caractéristiques des parcours en mois

\begin{tabular}{|l|c|c|}
\hline & FPT & FPE \\
\hline moyenne ou \% & & \\
\hline mois en emploi & 27 & 28 \\
\hline mois au chômage & 4 & 2,5 \\
\hline mois en inactivité & 1,3 & 1,9 \\
\hline mois en formation & 0,5 & 0,6 \\
\hline temps d'accès au premier emploi & 4,7 & 4,1 \\
\hline premier emploi privé & $29 \%$ & $24 \%$ \\
\hline premier emploi = emploi actuel & $43 \%$ & $36 \%$ \\
\hline temps d'accès au premier emploi si premier emploi = emploi actuel & 5 & 6,3 \\
\hline mois au chômage si premier emploi = emploi actuel & 4,5 & 2,5 \\
\hline
\end{tabular}

Lire : sur la période d'observation de 36 mois, les jeunes employés dans la FPT (fonction publique territoriale) sont en emploi 27 mois contre 28 dans la FPE (fonction publique d'État) ; $29 \%$ de ces jeunes employés dans la FPT ont connu un premier emploi dans le secteur privé contre $24 \%$ des jeunes employés dans la FPE. Dans la FPT, 43 fois sur cent l'emploi actuel est le premier emploi occupé contre $36 \%$ dans la FPE; si l'emploi actuel est identique au premier emploi, le temps d'accès au premier emploi est de 5 mois dans la FPT contre 6,3 dans la FPE.

Source : Enquête Génération 98, Céreq.

ceux de la FPE (tableau 4). Ces derniers sont souvent plus spécifiques et s'inscrivent dans la continuité de formations ad hoc. Dans le privé, la structure de la population active est d'environ deux tiers d'employés et d'ouvriers et $15 \%$ de cadres, structure que l'on retrouve dans la FPT et pas dans la FPE où, par exemple, les ouvriers sont largement sous-représentés. La plus grande proximité $\mathrm{FPT} /$ secteur privé s'exprime aussi en matière de modes de gestion de la main-d'œuvre, à travers la diversité des formes d'emploi utilisées (les emplois à statut, les hors statuts, les emplois aidés, le recours à la sous-traitance privée, le passage par des structures relais).

Concernant les statuts, contrairement aux employeurs du secteur privé, la FPT recrute plus de la moitié de ses nouveaux salariés sur des contrats temporaires (CDD) ou en utilisant largement les possibilités offertes par les politiques de l'emploi (emplois-jeunes, CES contrat emploi solidarité). Ainsi, trois ans après leur sortie du système éducatif, seulement un jeune sur cinq employé dans la FPT est fonctionnaire, la même proportion étant recrutée en CDI. Ils sont plus du double dans la FPE. Notons que les jeunes de la Génération 98 ont été recrutés davantage sur des emplois à durée déterminée dans les deux fonctions publiques que dans le secteur privé, qui ne recrute qu'un quart de ses salariés en CDD (tableau 5), la proportion étant d'un tiers pour l'ensemble des jeunes. Le poids des emplois-jeunes n'explique pas à lui seul le décalage entre public et privé, leurs parts respectives pour la FPE et la FPT étant de 28 et $20 \%$.

À cette importance des contrats à durée limitée correspond également un plus fort développement du temps partiel dans la FPT. Ainsi, le pourcentage de salariés à temps complet est supérieur de près de $10 \%$ dans la FPE par rapport à la FPT, qui compte $84 \%$ de travailleurs dans cette situation. Ces salariés à temps partiel sont pour $80 \%$ des femmes dans la FPE contre $90 \%$ pour la FPT, alors que le pourcentage de femmes est le même dans les deux fonctions publiques (près de $60 \%$ ). En d'autres termes, la FPT recrute davantage que la FPE pour des postes à temps partiel, majoritairement occupés par des femmes. Ce résultat descriptif est confirmé par la procédure logistique : la probabilité d'être recruté dans la FPT étant diminuée dés lors que le poste est à temps complet ${ }^{9}$.

\footnotetext{
${ }^{9}$ En revanche, alors que les femmes représentent le même pourcentage dans les deux fonctions publiques, la probabilité d'être, trois années après la date de fin d'études, dans la FPT, est augmentée par le fait d'être une femme «toutes choses égales par ailleurs ». Un tel résultat peut traduire, par exemple, le fait qu'à diplôme équivalent les femmes sont davantage recrutées dans la FPT que dans la FPE. Une telle conjecture nécessiterait néanmoins des investigations complémentaires sur un échantillon plus important, par région, type de certification, etc.
} 
Tableau 4

\section{Caractéristiques des emplois}

\begin{tabular}{|c|c|c|c|c|c|}
\hline$\%$ & FPT & FPE & $\%$ & FPT & FPE \\
\hline Statut & & & Temps de travail & & \\
\hline Fonctionnaire & 19 & 41 & Temps complet & 84 & 92 \\
\hline Contrat à durée indéterminée & 19 & 13 & Temps Partiel $80 \%$ & 4,2 & 1,9 \\
\hline Contrat à durée déterminée & 28 & 23 & TP $60 \%$ & 1,4 & 0,8 \\
\hline Contrat emploi solidarité & 4 & 2 & TP $50 \%$ & 7,5 & 4,3 \\
\hline Emploi-jeune & 28 & 20 & $\mathrm{TP}<50 \%$ & 3,2 & 1,4 \\
\hline Autres emplois aidés & 2 & 1 & & & \\
\hline \multicolumn{6}{|l|}{ Emplois occupés } \\
\hline Ingénieurs et cadres & 13 & 26 & $\begin{array}{l}\text { Aides-soignants, adjoints de } \\
\text { services hospitaliers }\end{array}$ & 4 & 1 \\
\hline Enseignants, formateurs & 4 & 34 & Pompiers, gendarmes, police & 7 & 14 \\
\hline $\begin{array}{l}\text { Assistantes sociales, animateurs, } \\
\text { conseillers familiaux }\end{array}$ & 21 & 2 & Employés administratifs & 1 & 1 \\
\hline $\begin{array}{l}\text { Professions intermédiaires des } \\
\text { collectivités territoriales et catégories B }\end{array}$ & 6 & 7 & $\begin{array}{l}\text { Personnels des services directs } \\
\text { aux particuliers }\end{array}$ & 2 & 1 \\
\hline Techniciens, Agents de maîtrise & 3 & 2 & Ouvriers Qualifiés (hors jardiniers) & 2 & 3 \\
\hline $\begin{array}{l}\text { Agents de constatation et de } \\
\text { recouvrement }\end{array}$ & 5 & 2 & Jardiniers & 6 & 1 \\
\hline Adjoints et agents administratifs & 11 & 5 & Ouvriers Non Qualifiés & 3 & 1 \\
\hline Agents de service & 12 & 2 & & & \\
\hline
\end{tabular}

Lire : La FPE compte $41 \%$ de fonctionnaires contre $19 \%$ dans la FPT.

Source : Enquête génération 98, Céreq.

Plus surprenante est la présence de nombreux salariés en CDI, alors que ce type de statut semble a priori utilisé de manière assez marginale et n'existe d'ailleurs pas en droit administratif ${ }^{10}$ ! Toutefois, comme cela a été évoqué plus haut, l'employeur FPT est appréhendé dans une acception large qui inclut des

10 Dans la FPT, les recrutements hors procédures statutaires (concours, fonctionnaires) pour des postes «contractuels» sont des CDD de 3 ans au plus, renouvelables ( $c f$. encadré 2). Les autres possibilités de CDI sont le droit d'option de salariés précédemment sous ce type de contrats (selon la circulaire Berkani, circulaire du Tribunal des conflits portant sur le fait que «les agents non titulaires de l'État, de ses établissements publics à caractère administratif et des établissements publics locaux d'enseignement ne sont normalement pas liés à leur employeur par un contrat de droit privé ») ou lors de la reprise par une collectivité de l'activité d'une association. De manière générale, au-delà des collectivités territoriales stricto sensu (communes, départements régions) et des établissements publics locaux, différents organismes public « locaux » ou à fonds publics peuvent recruter sur des contrats de droit privé donc des CDI (Desbarats, 2005). institutions sur fonds publics qui recourent aux contrats de droit privé, dont le CDI. La raison d'une présence importante de CDI tant dans la FPT que dans la FPE doit néanmoins vraisemblablement beaucoup à l'ambiguïté actuelle des statuts de nombreux salariés en CDD de droit public. Ainsi, un contrat très répandu est le « $3+3 »$, soit le contrat de trois ans renouvelable deux fois, donc pour une période en théorie maximale de neuf ans. Or, en réalité, la règle de droit qui conduit à ne renouveler les CDD que deux fois est issue du droit du travail ; elle s'applique donc aux contrats relevant du privé et entretient une relation ambiguë avec le droit administratif, d'où une pratique largement répandue de succession des $« 3+3$ » et même de contrats de plus courte durée. Rien d'étonnant alors à ce que ces salariés se déclarent en CDI. Dans le cas de la FPT, cette confusion est encore renforcée par les notions d'emplois permanents, donc à durée indéterminée. Cette ambiguité des statuts a été récemment soulevée, notamment par les instances européennes, 
Tableau 5

Profession et statut dans les deux fonctions publiques et dans le privé (en \%)

\begin{tabular}{|c|c|c|c|c|c|c|c|c|c|c|c|c|c|c|c|}
\hline & \multicolumn{2}{|c|}{ Cadres } & \multicolumn{2}{|c|}{$\begin{array}{l}\text { Enseignants } \\
\text { formateurs }\end{array}$} & \multicolumn{2}{|c|}{$\begin{array}{c}\text { Autres } \\
\text { professions } \\
\text { intermédiaires }\end{array}$} & \multicolumn{2}{|c|}{$\begin{array}{c}\text { Techniciens, } \\
\text { agents de } \\
\text { maîtrise }\end{array}$} & \multicolumn{2}{|c|}{ Employés } & \multicolumn{2}{|c|}{ Ouvriers } & \multicolumn{2}{|c|}{ Tous } & \multirow[t]{2}{*}{ Privé } \\
\hline & FPT & FPE & FPT & FPE & FPT & FPE & FPT & FPE & FPT & FPE & FPT & FPE & FPT & FPE & \\
\hline $\begin{array}{l}\text { Fonctionnaire } \\
+\mathrm{CDI}\end{array}$ & 38 & 77 & 24 & 40 & 33 & 59 & 27 & 64 & 44 & 48 & 39 & 47 & 38 & 53 & 76 \\
\hline $\begin{array}{l}\text { CDD dont } \\
\text { emplois-jeunes }\end{array}$ & 62 & 23 & 76 & 60 & 67 & 41 & 73 & 36 & 57 & 52 & 61 & 53 & 62 & 47 & 24 \\
\hline
\end{tabular}

Sigles : FPT : fonction publique territoriale ; FPE : fonction publique d'État ; CDI : contrat à durée indéterminée ; CDD : contrat à durée déterminée.

Source : Enquête Génération 98, Céreq.

qui ont sommé la France de mettre sa législation en conformité avec le droit communautaire. Ainsi, très récemment, la loi du 26 juillet 2005 a ouvert la voie au CDI de droit public, statut sous lequel passeront notamment des salariés sous contrat qui occupent des CDD depuis six ans au moins (majoritairement des $《 3+3 »){ }^{11}$. Le CDI succédera donc au « $3+3$ » et est vraisemblablement appelé à se développer.

Il est très important de noter que les différences de statuts constatées entre FPE et FPT ne sont pas le corollaire de structures d'emplois elles-mêmes différentes. Ainsi, s'il y a moins de fonctionnaires et de CDI au sein de la FPT, ce n'est pas parce que les catégories qui comptent le plus de fonctionnaires sont plus représentées dans la FPE, les différences s'observant au sein des catégories. Par exemple, les cadres (hors enseignants) de la FPT comptent $38 \%$ de fonctionnaires et CDI et $62 \%$ de CDD (dont emploisjeunes), contre respectivement $77 \%$ et $23 \%$ pour la FPE (tableau 5).

$\mathrm{Au}$ terme de cette première analyse, la FPT apparaît être effectivement un employeur spécifique en regard de la FPE, tant en ce qui concerne les modes de gestion de la main-d'œuvre (statut, temps de travail, mode d'accès à l'emploi) que les caractéristiques individuelles (âges, diplômes). Cette distinction étant faite, reste à mieux appréhender les différents éléments évoqués pour la seule FPT. C'est l'objectif de la section suivante.

${ }^{11}$ Loi du 26 juillet 2005 portant diverses mesures de transposition du droit communautaire de la fonction publique.

\section{QUELS DÉTERMINANTS DES SALAIRES ET DU STATUT DANS LA FONCTION PUBLIQUE TERRITORIALE ?}

Les résultats précédents mettent en exergue les spécificités de la FPT en regard de la FPE. Ces deux ensembles n'ont manifestement pas des modes de gestions de la main-d'œuvre similaires. Par ailleurs, la FPT semble posséder des spécificités propres. La section suivante tente d'éclairer ces modes de gestions de la FPT selon deux approches liées. La première consiste à étudier les déterminants des salaires des jeunes essentiellement en fonction du type de diplôme détenu et du statut. La seconde explicite le recours aux différents statuts au sein de la FPT en fonction du parcours et du type d'emploi occupé.

\section{Une prime aux diplômes professionnels?}

Les estimations pour les salaires sont effectuées en utilisant une fonction de gains mettant en relation le salaire horaire avec le sexe, le diplôme, le statut, un premier emploi dans le secteur privé (tableau 6).

À statut et caractéristiques individuelles égales, le fait d'être une femme diminue le salaire de $5 \%$. L'expérience étant calculée en fonction des durées exactes des séquences d'emplois, la différence n'est pas liée, comme cela est souvent évoqué, à des périodes d'inactivité. L'écart de salaire entre hommes et femmes « toutes choses égales par ailleurs » demeure inférieur 
à celui observé pour l'ensemble de la population active, notamment par Meurs et Ponthieux (1999), qui obtiennent un écart salarial au minimum de $7 \%{ }^{12}$.

Pour les diplômes, deux principaux constats sont effectués : un sort relativement favorable aux nondiplômés, une prime aux diplômes professionnels. En effet, si les moins diplômés sont relativement nombreux au sein de la FPT, il n'existe pas de différenciations salariales nettes entre les niveaux de formation (inférieurs au baccalauréat). De fait, les détenteurs de $\mathrm{CAP}$ et BEP ne sont pas rémunérés davantage que les salariés non qualifiés, et même relativement moins. Ce résultat surprenant pourrait s'expliquer par des parcours qualifiants extrascolaires effectués par les sortants non diplômés. Les petits boulots, les activités associatives ou sportives, par exemple, leur ont permis de constituer des compétences ou une expérience monnayées ensuite sur le marché du travail.

Les rendements des baccalauréats traduisent clairement une prime aux diplômes techniques ou professionnels par rapport à ceux de la filière générale, avec un écart de près de $5 \%$ pour les baccalauréats techniques en regard des baccalauréats généraux. On retrouve cette «prime au technique» pour le niveau 3. Toutefois, celle-ci concerne uniquement les BTS et ne touche pas les DUT. Deux explications vraisemblables sont envisageables : les DUT ont un contenu plus général et/ou les spécificités techniques requises au sein de la FPT relèvent davantage des BTS que des DUT. Ces deux assertions doivent évidemment faire l'objet d'investigations plus spécifiques pour être validées.

Pour les niveaux supérieurs, les bacs +3 n'ont pas de rendements significativement supérieurs aux BTS (seulement $+2 \%$ ), ce qui traduit à nouveau vraisemblablement une prime au technique, tandis que les bacs +4 permettent d'accroître le salaire de $5 \%$ par rapport à la catégorie précédente. Les rendements les plus élevés vont néanmoins aux bac +5 qui sont en majorité des DESS - diplôme d'études supérieures spécialisés (Lopez, 2002), soit encore des diplômes professionnalisants.

Un début de carrière dans le privé permet d'obtenir un salaire relatif supérieur de $3 \%$ «toutes choses égales par ailleurs ». La FPT semble donc en partie

${ }^{12}$ Ceci même si l'on ajoute les régions et les emplois comme variables explicatives. être sur un marché du travail où ses salariés potentiels sont en concurrence avec ceux du privé. Un ajustement du salaire aux tarifs du marché pour se procurer des compétences rares ou recherchées serait pratiqué. Une rémunération de l'expérience antérieure dans le secteur privé peut également expliquer ce différentiel.

\section{Des statuts liés au niveau d'études et à l'emploi occupé}

Pour les statuts des jeunes, les premiers constats concernent les salaires. Ainsi, occuper un contrat à durée indéterminée est quasiment équivalent au fait d'être fonctionnaire en termes de salaire trois ans après la date de fin d'études (tableau 6). En revanche, être en CDD diminue le salaire de près de $10 \%$ et de plus de $25 \%$ pour les emplois-jeunes et les contrats emploi-solidarité, à diplôme égal notamment.

Il est important de noter que ces différences de salaires sont davantage liées au type de contrat de travail qu'à l'emploi occupé. En effet, comme le montrent les investigations qui suivent, les statuts recouvrent des qualifications diverses. Par exemple, les emploisjeunes concernent à la fois des emplois non qualifiés et des professions intermédiaires (tableau 8) ${ }^{13}$.

Le constat d'une égalité entre un salarié en CDI et un fonctionnaire est relativisé selon le niveau de formation, même si la distribution des jeunes par niveau n'est pas toujours nettement différenciée entre les divers statuts (tableau 7). Il est intéressant alors d'observer l'impact du statut par niveau de certification. Les régressions ne sont pas reproduites ici; elles sont identiques à celle effectuée dans le tableau $\mathbf{6}$, à l'exception évidente des diplômes non concernés.

Les principaux résultats sont les suivants : occuper un emploi-jeune est d'autant plus pénalisant que le niveau de certification est élevé, l'ensemble de ces emplois étant rémunérés au niveau du SMIC (salaire minimum interprofessionnel de croissance) alors que plus de $20 \%$ des emplois-jeunes détiennent une certification supérieure à bac $+2(25 \%$ de salaire en moins par rapport aux fonctionnaires pour un niveau

\footnotetext{
13 De fait, une estimation d'une fonction de gains intégrant les variables d'emploi (PCS - profession et catégorie sociale - du tableau 8) change peu les coefficients des variables relatives au statut d'emploi.
} 
Tableau 6

Déterminants des salaires dans la fonction publique territoriale

\begin{tabular}{|l|c|}
\hline \multicolumn{1}{|c|}{ Variable expliquée Logarithme du salaire net horaire : primes incluses (1) } & Rendement salarial \% \\
\hline Femme & $-5^{(2)}$ \\
\hline Diplômes & réf. \\
\hline non diplômés & -2 \\
\hline CAP-BEP non diplômés & -3 \\
\hline CAP & 1 \\
\hline BEP & +3 \\
\hline Bac. non diplômé & +5 \\
\hline Bac Pro. & 0 \\
\hline Bac. Techno. & +5 \\
\hline Bac général-DEUG non Dipl. & +7 \\
\hline BTS-DUT non Dipl. & $+10(4)$ \\
\hline DEUG & +7 \\
\hline BTS & +9 \\
\hline DUT & +14 \\
\hline Bac. + 3 & +36 \\
\hline Bac. + 4 & +3 \\
\hline Bac. + 5 & \\
\hline Premier emploi privé & réf. \\
\hline Statuł & 0 \\
\hline Fonctionnaire & -9 \\
\hline Contrat à durée indéterminée & -24 \\
\hline Contrat à durée déterminée & -25 \\
\hline Contrat emploi solidarité & $-0,50$ \\
\hline Emploi-Jeunes & 36 \\
\hline Autres emplois aidés & 1570 \\
\hline \% de variance des salaires expliquées R2 (5) & \\
\hline Effectif & \\
\hline
\end{tabular}

(1) les variables relatives au parcours (expérience, durée de chômage, temps d'accès au premier emploi, ancienneté dans le dernier emploi) et au temps de travail sont également prises en compte, ainsi que pour les diplômes : écoles et prépa et IUFM (Institut universitaire de formation des maîtres) non reproduits car marginaux.

La fonction de gains FG est une régression linéaire du logarithme du salaire sur un ensemble de variables. Le recours au logarithme permet de mesurer directement l'effet d'une unité supplémentaire de chaque variable sur le salaire (ici coefficients X par 100 pour obtenir des \%).

(2) Lire : les femmes gagnent en moyenne $5 \%$ de moins que les hommes.

(3) pour la valeur 0 , les coefficients sont non significatifs au seuil de $1 \%$, ce qui s'explique toujours par un coefficient très proche de 0 , soit un faible écart à la référence.

(4) Lire : les détenteurs d'un BTS perçoivent des salaires supérieurs de $10 \%$ à ceux des non-diplômés (réf.).

(5) Signifie que $36 \%$ de la variance des salaires s'explique par l'ensemble des variables retenues; reste donc $74 \%$ expliqués par des variables inobservables (aptitudes intrinsèques, par exemple) ou inobservées ici. Ce résultat est du même ordre de grandeur que celui obtenu dans la plupart des études. Source : Enquête Génération 98, Céreq, calculs des auteurs.

inférieur au baccalauréat, contre $34 \%$ pour un niveau supérieur à bac +2).

Le CDD a un impact identique pour les bac +2 , soit un salaire inférieur d'environ $15 \%$ par rapport à celui des fonctionnaires. En revanche, ce statut est peu pénalisant pour les détenteurs d'un diplôme supérieur à bac +2 qui représentent près d'un tiers des salariés en CDD. La situation est inversée pour le CDI. Ce statut est pénalisant, notamment pour les diplômes supérieurs au baccalauréat (respectivement 8 et $4 \%$ 
Tableau 7

Type d'emplois et niveaux de diplômes des salariés de la fonction publique territoriale

\begin{tabular}{|l|c|c|c|c|}
\hline \multicolumn{1}{|c|}{$\%$} & Fonctionnaire & CDI & CDD & Emploi-Jeune \\
\hline tous niveaux* & 20 & 20 & 28 & 28 \\
\hline$<$ Bac & 18 & 35 & 32 & 23 \\
\hline Bac & 29 & 30 & 24 & 34 \\
\hline Bac +2 & 21 & 22 & 14 & 21 \\
\hline$>$ Bac & 32 & 13 & 30 & 22 \\
\hline Total & 100 & 100 & 100 & 100 \\
\hline
\end{tabular}

Lire : $18 \%$ des jeunes employés dans la FPT ayant le statut de fonctionnaire en 2001 ont un niveau d'études inférieur au bac. $*+4 \%$ CES (contrat emploi solidarité) pour obtenir $100 \%$.

Source : Enquête Génération 98, Céreq.

pour les bacheliers en regard des fonctionnaires). En revanche, le CDI procure un avantage salarial pour ceux dont le niveau d'études est inférieur au baccalauréat (+ $5 \%$ de salaire par rapport aux fonctionnaires).

Plusieurs hypothèses interprétatives peuvent être formulées à partir de ces résultats et des pratiques. De fait, si l'on se réfère aux modalités «légales » de recrutement, le CDI ne peut être utilisé que par certaines structures recrutant des salariés sous contrat de droit privé ou pour des cas très particuliers. Ces structures peuvent appliquer des conventions plus avantageuses, offrir de meilleurs salaires, ces emplois étant aussi souvent «techniques». Tout d'abord, le CDI est susceptible d'être utilisé pour attirer des individus peu diplômés, mais ayant des qualifications recherchées, ou bien acceptant des emplois pénibles ou contraignants, la rémunération offerte étant supérieure à celle issue des grilles de salaire des fonctionnaires de la FPT. Ensuite, les CDD sont aussi utilisés pour les plus diplômés. Dans ce cas, on constate une égalité de traitement avec les fonctionnaires. Ces CDD permettent de capter directement une main-d'œuvre très qualifiée pour des missions de court ou moyen terme. Les raisons de ce recours sont nombreuses et de nature très différente : main-d'œuvre non disponible par la voie des concours ou moyen de s'affranchir de la procédure, recours à des personnels contractuels plus flexibles, souhait de jeunes diplômés d'être recrutés hors cadres statutaires considérés comme peu valorisants, utilisation des formes particulières d'emploi proposés dans le cadre des politiques d'emploi.

Une étude des déterminants de l'appartenance aux différents statuts, via un modèle logistique multinomial, permet de préciser les assertions précédentes, la catégorie de référence étant celle des fonctionnaires (tableau 8).

Plus le nombre de mois en chômage est important, plus la probabilité d'occuper un emploi-jeune ou un CDD est forte ; ces statuts sont effectivement et logiquement souvent des choix de la seconde chance. Si le chômage n'a pas d'impact significatif sur l'accès à un CDI, le temps d'accès au premier emploi a un impact positif (référence fonctionnaires). En d'autres termes, l'accès au statut de fonctionnaire est plus rapide que l'accès à un CDI. De fait, les salariés en CDI de la FPT ont occupé plus souvent d'autres emplois avant d'obtenir ce statut (impact négatif de la variable premier emploi égal emploi actuel). Par rapport au statut de fonctionnaire, le fait d'être une femme diminue fortement la probabilité d'être en emploi-jeune et en CDI.

De fait, les femmes sont davantage représentées que les hommes dans les statuts de fonctionnaires (25\% des femmes contre $15 \%$ des hommes). Le statut étant en partie lié à l'emploi occupé, vingt variables dichotomiques représentant plusieurs professions agrégées ont donc été intégrées à la spécification du modèle. Ces variables fournissent des indications sur les professions pour lesquelles la FPT a davantage utilisé tel ou tel statut. Toutefois, cette répartition des effectifs entre les catégories est moins équilibrée que celle relative aux dix-sept diplômes. Les commentaires sont donc globaux ou bien portent sur des catégories dont les effectifs ont été jugés suffisants.

La probabilité d'être en emploi-jeune, plutôt que sous un autre statut, est relativement élevée pour les professions «formateurs, » « assistantes sociales, animateurs et conseillers familiaux », « techniciens et agents 
Tableau 8

\section{Les déterminants du statut}

\begin{tabular}{|c|c|c|c|}
\hline Référence fonctionnaire & Emploi-jeune & CDD & CDI \\
\hline \multicolumn{4}{|l|}{ Parcours } \\
\hline mois en emploi & ++ & 0 & +++ \\
\hline mois au chômage & ++ & ++ & 0 \\
\hline mois en inactivité & + & ++ & 0 \\
\hline mois en formation & + & ++ & 0 \\
\hline mois en études & - & - & - \\
\hline temps d'accès au premier emploi & 0 & - & + \\
\hline Femme & --- & - & --- \\
\hline Premier emploi privé & +++ & 0 & + \\
\hline Premier emploi $=$ emploi actuel & -- & - & --- \\
\hline \multicolumn{4}{|l|}{ Emplois occupés } \\
\hline Ingénieurs et cadres & réf. & réf. & réf. \\
\hline Personnel administratif supérieur des collectivités locales & -- & - & 0 \\
\hline Enseignants, formateurs & +++ & 0 & ++ \\
\hline Sages femmes - techniciens médicaux & 0 & 0 & +++ \\
\hline Assistantes sociales, animateurs, conseillers familiaux. & ++ & - & + \\
\hline Professions intermédiaires des collectivités territoriales et catégories B & 0 & -- & - \\
\hline Maîtrise administrative & - & --- & -- \\
\hline Techniciens, agents de maîtrise & ++ & -- & 0 \\
\hline Agents de constatation et de recouvrement & -- & -- & 0 \\
\hline Adjoints et agents administratifs & ++ & -- & 0 \\
\hline Agents de service & 0 & - & ++ \\
\hline Aides-soignants, adjoints de services hospitaliers & -- & -- & ++ \\
\hline Pompiers, gendarmes, police & -- & --- & - \\
\hline Employés administratifs & 0 & --- & --- \\
\hline Personnels des services directs aux particuliers & 0 & -- & ++ \\
\hline Ouvriers Qualifiés (hors jardiniers) & - & --- & + \\
\hline Jardiniers & +++ & 0 & 0 \\
\hline Ouvriers Non Qualifiés & 0 & --- & - \\
\hline Variance expliquée (Pseudo R² : Cox et Snell) 35,8 \% (*) & & & \\
\hline
\end{tabular}

Lire : 1 mois de chômage augmente la probabilité d'occuper un emploi-jeune deux fois plus qu'un mois d'inactivité, par rapport au statut fonctionnaire.

Lire : Les professions «Assistantes sociales, animateurs, conseil familiaux » et « Ouvriers Non Qualifiés » ont la probabilité la plus élevée d'occuper un emploi-jeune par rapport aux ingénieurs et cadres, supérieure de un tiers à celle des techniciens et agents de maîtrise.

(*) Signifie que 35,8\% de la variance des salaires s'explique par l'ensemble des variables retenues, reste donc 74,2 \% expliqués par des variables inobservables (aptitudes intrinsèques, par exemple) ou inobservées ici. Ce résultat est du même ordre de grandeur que celui obtenu dans la plupart des études.

Source : Enquête génération 98, Céreq, calculs des auteurs.

de maîtrise » et « jardiniers ». Or, ce constat est réalisé en fonction d'une catégorie de référence (les cadres et ingénieurs) où les effectifs relatifs en emplois-jeunes sont parmi les plus bas. Ceci n'est pas le cas pour les $\mathrm{CDD}$, ces derniers étant relativement nombreux pour les plus qualifiés. Les résultats précédents suggèrent que pour ces emplois qualifiés, le CDD ne joue pas le même rôle que les emplois-jeunes, puisque le CDD n'est pas pénalisant du point de vue salarial pour les plus diplômés. En d'autres termes, si le CDD peut 
permettre de recruter une main-d'œuvre qualifiée en dehors des grilles de fonctionnaires, l'emploi-jeune conduit majoritairement à un déclassement salarial.

Les quatre catégories d'emplois qui augmentent le plus la probabilité d'être en CDI sont notamment les personnels des services directs aux particuliers, les agents de services, les aides-soignants et adjoints de services hospitaliers. Une explication envisageable, liée aux résultats précédents, est que les moins diplômés qui occupent ces emplois sont recrutés via des structures qui utilisent de tels contrats et que cette situation est plutôt favorable en termes de salaire.

Nos investigations invitent à des prolongements afin de préciser les logiques de recrutement et de gestion des carrières au sein de la FPT. Ceci d'autant que les effectifs n'ont pas permis de découpages territoriaux et des études fines par type d'emplois, d'une part. D'autre part, si la fonction publique territoriale a été étudiée comme un ensemble à part entière, c'est-àdire en la séparant de la FPE, les administrations qui la constituent (régions, départements, communes) n'ont pu être analysées séparément. Des études plus ciblées et portant sur des parcours plus longs doivent donc compléter cette analyse.

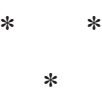

L'observation des sortants du système éducatif de 1998 ayant un employeur public en 2001 confirme la spécificité des emplois et des modes de gestion de la fonction publique territoriale au sein de l'ensemble de la fonction publique.

L'utilisation d'une diversité de modes de gestion (statuts, salaires, profils) selon les catégories d'emplois et les compétences recherchées, la prime aux diplômes techniques, la prise en compte de l'expérience dans le secteur privé, soulignent l'inscription de la FPT dans des modes de gestion proches du secteur marchand. À l'opposé, la relative protection des femmes et des sans-diplômes, l'importance des recrutements dans le cadre des politiques d'emploi (les emplois-jeunes principalement) renvoient à une gestion publique des emplois. La FPT apparaît donc comme un employeur partagé entre gestion publique et gestion marchande.

La gestion de la fonction publique territoriale se caractérise aussi par une utilisation très importante de contractuels. Or, le recours significatif aux contrats hors statut peut contribuer à la hausse de la précarité sur le marché du travail et à une insécurité croissante dans l'emploi, y compris pour les salariés qui ont plusieurs années d'activité dans la fonction publique. Ce mouvement s'apparente aux observations réalisées dans le secteur privé incluant les salariés en CDI (Béhagel, 2003).

Par ailleurs, pour l'ensemble de la fonction publique, le recours aux salariés hors statut de fonctionnaire est appelé à se développer, compte tenu notamment de la mise en conformité de la législation française avec le droit communautaire concernant les CDD. Dans ce domaine, toute la question est de savoir si l'augmentation des salariés sous contrat dans la fonction publique, via la FPT, et la législation vont accroître l'insécurité dans l'emploi. La législation actuelle est, en effet, relativement pauvre, notamment concernant les CDI de droit public ; l'accroissement ou la réduction de l'insécurité dans l'emploi liée à l'extension de nouvelles formes de contrats de droit privé ne pourront donc être analysées qu'en fonction des évolutions législatives.

En réalité, si en moyenne l'insécurité dans l'emploi est plus forte au sein de la FPT en termes de nature de contrat, la situation est assez contrastée en regard des salaires. Le recours à d'autres statuts que celui de fonctionnaire est, en effet, aussi motivé par un souci d'efficacité qui bénéficie manifestement, au moins à court terme, directement au salarié. Par exemple, le recrutement de salariés hors statut de fonctionnaire permet d'offrir des salaires plus élevés que ceux de la grille salariale des fonctionnaires pour les individus dont le niveau d'études est inférieur au baccalauréat, salariés majoritairement représentés dans la FPT.

Face aux transformations à mener pour moderniser et améliorer la gestion des recrutements et des emplois dans la fonction publique, la fonction publique territoriale est considérée par certains comme «un modèle dans le processus de modernisation de la gestion des RH (ressources humaines) dans le secteur public » (Bourdon, 2004), dans la mesure où elle tente d'adapter le statut à des réalités de gestion de métiers et d'emplois très divers.

En définitive, la FPT apparaît comme le résultat d'une synthèse entre le modèle de la fonction publique d'État et le pouvoir des autorités territoriales, entre le système de statut général des fonctionnaires et les modes de gestion marchande des personnels. 


\section{Bibliographie}

Audier F. (1997), «La fonction publique: un débouché majeur pour les plus diplômés », Économie et Statistique, n 304-305, pp. 137-148.

Behagel L. (2003), «Insécurité de l'emploi : le rôle protecteur de l'ancienneté a-t-il baissé en France ? », Économie et Statistique, n 366, pp. 3-23.

Bourdon J. (2004), «La FPT, 20 ans d'évolution permanente », AJDA, ${ }^{\circ} 3$

Desbarats I. et Kopel S. (2005), «Les agents de la fonction publique et territoriale. De la précarité juridique à l'atout managérial?", Revue Française d'Administration Publique.

Di Paola V., Moullet S. (2003), «L'emploi public et les trajectoires d'insertion des jeunes », Économie et Statistique, ${ }^{\circ}{ }^{\circ}$ 369-370, pp. 49-74.

Doeringer P., Piore M. (1971), "Internal labor markets and manpower analysis", Lexington Mass, Heath Massachusets.

Germe J.-F., Pottier F. et Monchatre S. (2003), Les mobilités professionnelles : de l'instabilité dans l'emploi à la gestion des trajectoires, La Documentation française.
Lefresne F., Fondeur Y. (2000), « Les jeunes, vecteur de la transformation structurelle des normes d'emploi en Europe?», Travail et Emploi, $\mathrm{n}{ }^{\circ} 83$, juillet, pp. 115- 136.

Le Lidec P., de Montricher N. (2004), Décentraliser et gérer, analyse rétrospective et prospective de l'emploi public dans les collectivités territoriales, La Documentation française, Paris.

Lichtenberger Y. (1999), « Compétence, organisation $\mathrm{du}$ travail et confrontation sociale », FormationEmploi, $\mathrm{n}{ }^{\circ} 67$, pp. 93-107.

Lopez A. (2002), «Le parcours des jeunes diplômés entrant dans la fonction publique » in Comptes rendu des journées d'étude, «La fonction publique face au défi démographique : comment recruter demain ?», avril.

Meurs D. et Ponthieux S. (1999), «Emploi et salaires : les inégalités entre hommes et femmes en mars 98 », Premières synthèses, Dares 99.08, n 32.2 .

Raynaud Ph. (2003), « L'emploi public est tiré par la fonction publique territoriale », Économie et Statistique, $\mathrm{n}^{\circ}$ 369-370, pp. 75-92. 


\section{Résumé}

\section{Jeunes dans la fonction publique territoriale : entre gestion publique et marchande}

Françoise Dauty et Philippe Lemistre

La fonction publique territoriale est un employeur méconnu et pourtant dynamique. Comment ses recrutements influencent-ils les premières années de vie active des jeunes? Dans le cadre de l'observation de jeunes sortants du système éducatif, la fonction publique territoriale apparaît comme un employeur partagé entre gestion publique et gestion marchande. L'utilisation d'une diversité de modes de gestion selon les catégories d'emplois, la prime aux diplômes techniques, la prise en compte de l'expérience hors secteur public soulignent l'inscription de la fonction publique territoriale dans des modes de gestion du privé. À l'opposé, la relative protection des femmes et des sans-diplômes, l'importance des recrutements dans le cadre des politiques d'emploi renvoient à une gestion publique.

\section{Mots Clés}

Recrutement, jeune, fonction publique territoriale.

Journal of Economic Literature: J 45, J 23 